\title{
Analysis of 13 Y-chromosomal STRs in an Arab population sample from Syria
}

\author{
Louai Abdin ${ }^{\mathrm{a}, \mathrm{b}}$, Koji Dewa ${ }^{\mathrm{b}, \mathrm{c}}$, Steven Rand ${ }^{\mathrm{b}}$, \\ Carsten Hohoff ${ }^{\mathrm{b}}$, Bernd Brinkmann ${ }^{\mathrm{b}, *}$ \\ ${ }^{a}$ Department of Legal Medicine, University of Tischreen, Syria \\ ${ }^{\mathrm{b}}$ Institut für Rechtsmedizin, Universität Münster Röntgenstr. 23, D-48149 Münster, Germany \\ ${ }^{\mathrm{c}}$ Department of Legal Medicine, Niigata University School of Medicine, Niigata, Japan
}

\begin{abstract}
Analysis of Y-chromosomal STRs has become increasingly important for forensic and population genetics. Although many populations have already been analysed, there are only few data on Arabic populations. A total of 13 polymorphic STR loci on the Y-chromosome were analysed in a Syrian population sample of 113 unrelated males (the majority from Lattakia) and 108 different haplotypes were observed, 104 haplotypes of them were found to be unique and the others were shared by two or three persons. The haplotype diversity was 0.9902 .

(C) 2003 Elsevier Science B.V. All rights reserved.
\end{abstract}

Keywords: Y-STR; Multiplex PCR; Population study; Y-haplotype

\section{Introduction}

Y-chromosomal short tandem repeat (STR) polymorphisms are increasingly being used in forensic genetics. Numerous populations have already been investigated, while there is a lack of information regarding Arabian populations. Therefore, we have analysed 113 unrelated males from Syria (the majority from Lattakia) at 13 Y STR loci (i.e., the loci known to determine the extended haplotype in the Y-STR Haplotype Reference Database (YHRD, http://ystr.charite.de) in addition to DXYS156, DYS437, DYS438, DYS439).

\footnotetext{
* Corresponding author. Tel.: +49-251-8355160; fax: +49-251-8355158.
}

E-mail address: brinkma@uni-muenster.de (B. Brinkmann). 

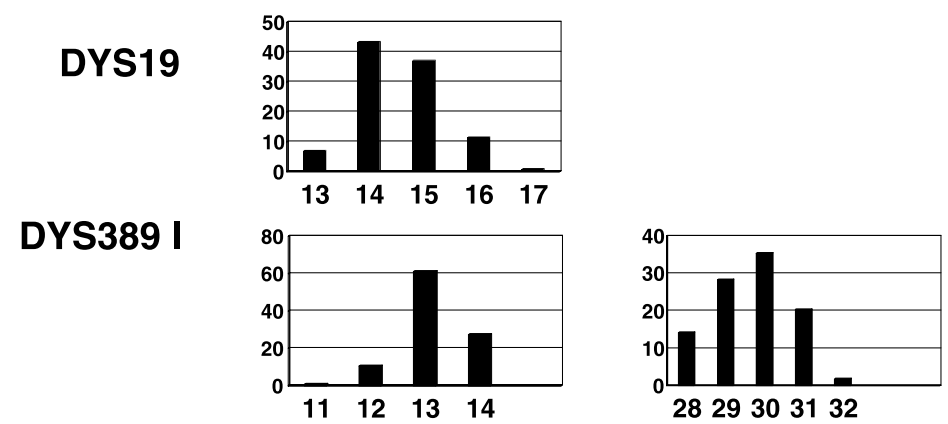

DYS389 ||

DYS390
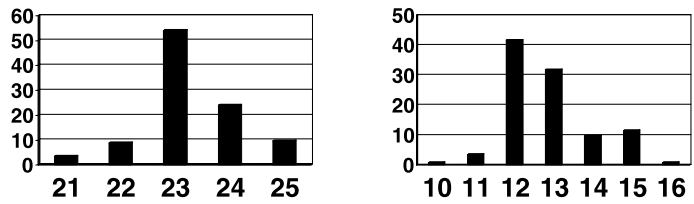

DYS393

DYS437
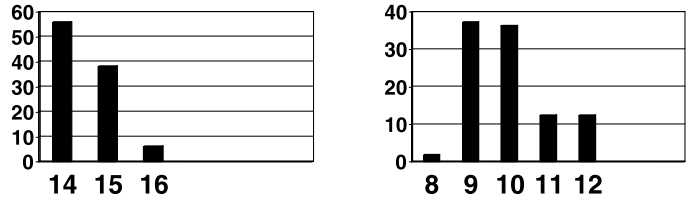

DYS438

DYS385

20
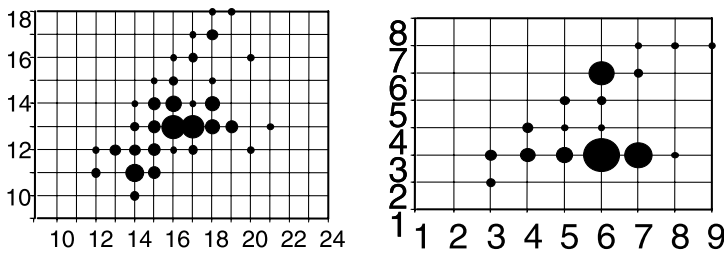

YCA II

20

DYS391
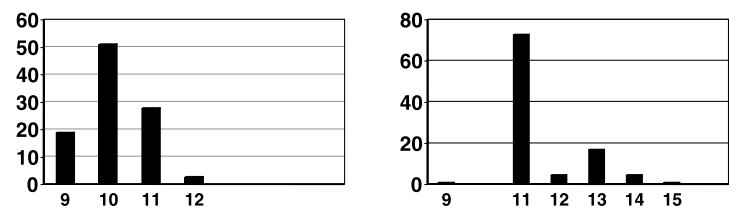

DYS392

DXYS156-Y

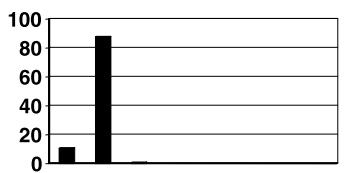

11.112 .1

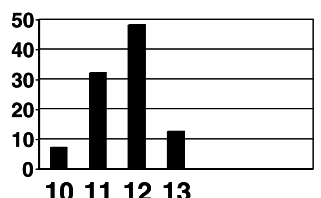

DYS439

Fig. 1. Allele frequencies [\%] in the Syrian population. 


\section{Materials and methods}

Genomic DNA was extracted from saliva swabs taken from 113 unrelated males living mainly in Lattakia using the Proteinase K/Chelex method. The Y-STRs were amplified in three multiplex PCR reactions: DYS19 DYS389 I and II, DYS390, DYS393 (primer sequences according to Ref. [1]), DYS385, DYS437, DYS438, DYS439 [2],DYS391 [3], DYS392 [4], DXYS156, YCA II [5] and amelogenin (Y-multiplex III). PCR fragments were analyzed by denaturing capillary electrophoresis on an ABI PRISM 310 Genetic Analyzer (Applied Biosystems) using a self-constructed sequenced allelic ladder. The frequencies of compound haplotypes were determined.

\section{Results and discussion}

The allele frequencies in the 13 Y-STRs in the Syrian population are shown in Fig. 1. Most systems display a unimodal allele distribution, while, e.g., at DYS392 allele 11 is predominant. The locus diversity values range from 0.22 (DXYS156-Y) up to 0.93 (DYS385). Regarding the YHRD 8-locus, 'minimal' haplotype 101 different haplotypes were observed (haplotype diversity, $D=0.9883$ ). Addition of the dinucleotide marker YCAII leads the 'extended' haplotype that was found 101 times $(D=0.9886)$. Adding another four markers (i.e., DXYS156-Y, DYS437, DYS438, DYS439) yields the 'extraextended' haplotype composed of 13 Y-STRs that was observed in 108 different haplotypes $(D=0.9902)$ of which 104 haplotypes were found to be unique and the others were shared by two or three individuals. Comparing the Syrian samples on the basis of the extra-extended haplotype with 268 Germans and 119 Vietnamese leads to 1 and 0 match, respectively. Comparison on the basis of the extended haplotype yields 18 matches in the YHRD. It remains to be elucidated if these sample an identical haplotype after having typed 13 Y-STRs. In conclusion, typing of 13 Y-STRs has lead to an informative, highly resolved database of the Syrian population.

\section{References}

[1] P. de Knijff, M. Kayser, A. Caglia, D. Corach, N. Fretwell, C. Gehrig, et al., Reference tables to: evaluation of Y-chromosomal STRs: a multicenter study (Kayser et al.) and Chromosome Y microsatellites: population genetic and evolutionary aspects (de Knijff et al.), Int. J. Leg. Med. 110 (1997) 141-149.

[2] P. Grignani, G. Peloso, P. Fattorini, C. Previdere, Highly informative Y-chromosomal haplotypes by the addition of three new STRs DYS437, DYS438 and DYS439, Int. J. Leg. Med. 114 (2000) 125-129.

[3] L. Gusmao, A. Gonzalez-Neira, P. Sanchez-Diz, M.V. Lareu, A. Amorim, A. Carracedo, Alternative primers for DYS391 typing: advantages of their application to forensic genetics, Forensic Sci. Int. 112 (2000) 49-57.

[4] M.G. Thomas, N. Bradman, H.M. Flinn, High throughput analysis of 10 microsatellite and 11 diallelic polymorphisms on the human Y-chromosome, Hum. Genet. 105 (1999) 577-581.

[5] K. Dewa, N.Q. Tuyen, S. Rand, C. Hohoff, B. Brinkmann, 13 Y-chromosomal STRs in a Vietnamese population, ICS 1239 (2003) 315-317. 